\title{
CORRESPONDENCE
}

\section{Effects of eplerenone on nephrotic syndrome in a patient with renovascular hypertension}

\author{
Hypertension Research (2011) 34, 404-406; doi:10.1038/hr.2010.249; published online 16 December 2010
}

Nephrotic syndrome accompanying renovascular hypertension $(\mathrm{RVH})$ is an uncommon condition. Successful reduction of massive proteinuria by nephrectomy, treatment with angiotensin-converting enzyme inhibitors (ACEIs) and/or angiotensin II receptor blockers (ARBs), and/or renal angioplasty have occasionally been reported. The use of these treatments suggests that activation of the renin-angiotensin-aldosterone (RAA) system may have an important role in this pathophysiology. Herein, we report a patient with nephrotic syndrome who did not fully respond to treatment with an ARB. Add-on therapy with a selective mineralocorticoid receptor (MR) blocker, eplerenone, resulted in the successful reduction of proteinuria.

A 61-year-old Japanese man with systemic hypertension was referred to our hospital for evaluation of severe hypertension and massive proteinuria. The patient's hypertension had been treated with benidipine $(8 \mathrm{mg}$ per day) since the age of 52 years. The systemic blood pressure had gradually increased to 170-200/90-110 mm Hg for 3 months despite additional treatment with amlodipine $(5 \mathrm{mg}$ per day) and doxazosin (4 mg per day). The patient had developed pretibial pitting edema, and laboratory examination revealed complications of nephrotic syndrome, showing proteinuria ( $3.6 \mathrm{~g}$ per day), hypoalbuminemia $(1.9 \mathrm{~g}$ per $100 \mathrm{ml})$, hypercholesterolemia $(315 \mathrm{mg}$ per $100 \mathrm{ml})$, hypokalemia (2.5$2.6 \mathrm{mEql}^{-1}$ ) and moderate renal insufficiency (serum creatinine, $1.18 \mathrm{mg}$ per $100 \mathrm{ml} ; 24-\mathrm{h}$ creatinine clearance, $46 \mathrm{ml} \mathrm{min}^{-1}$ ). Urine occult blood and urine glucose were negative, but hyaline casts (7 per whole field) and granular casts (4 per whole field) were observed in the urinary sediments. The selectivity index calculated according to the Cameron and Blandford method was 0.14, indicating that proteinuria was moderately selective. ${ }^{1}$ The plasma renin activity and the
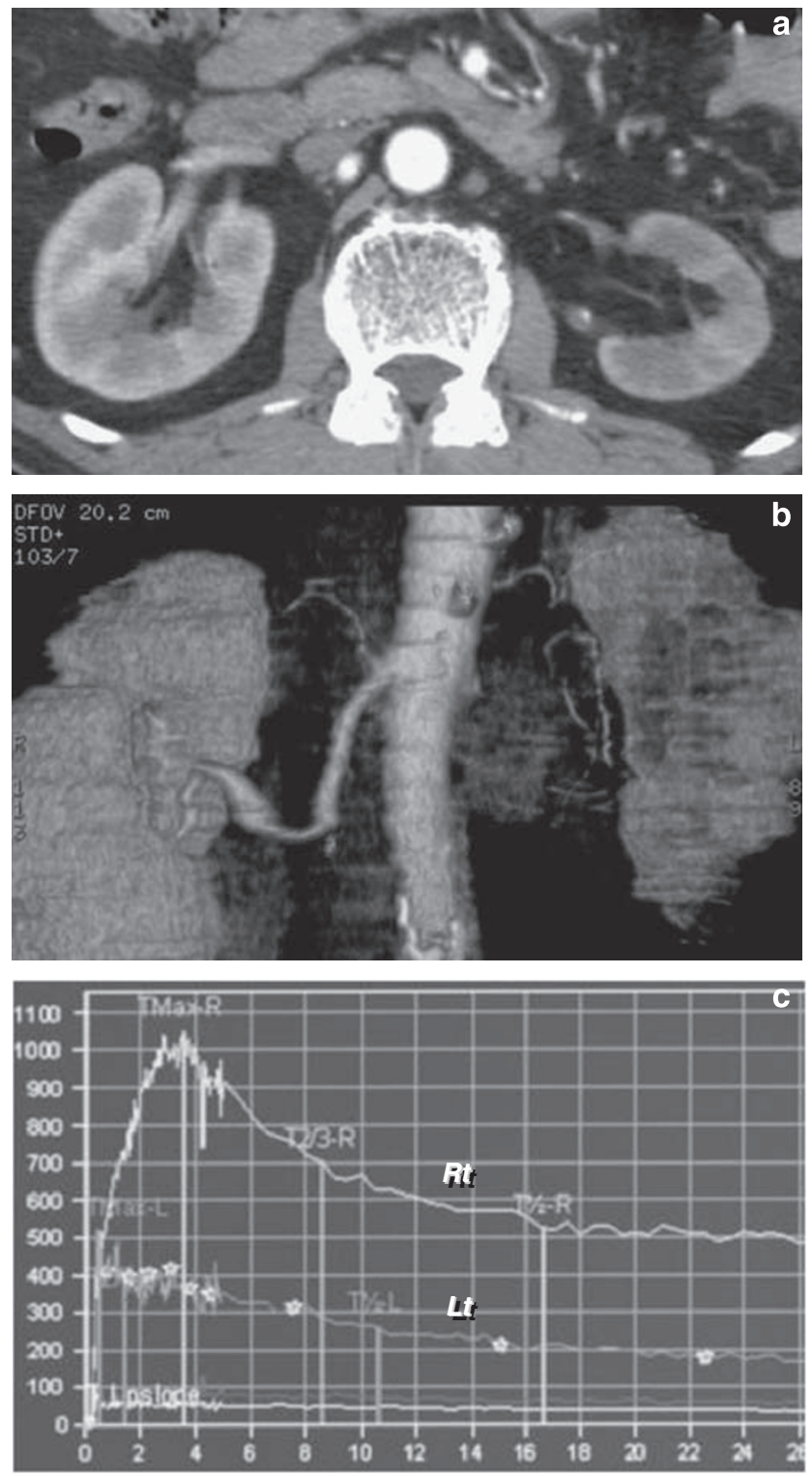

Figure 1 Radiological findings: (a) Computed tomography revealed marked atrophy of the left kidney. (b) Computed tomography-angiography showed occlusion of the left renal artery. (c) The 99mTcmercaptoacetyltriglycine renography demonstrated a functional defect of the left kidney due to the loss of renal arterial flow. 
aldosterone concentration were found to be high (>20 $\mathrm{ng} \mathrm{ml}^{-1} \mathrm{~h}^{-1}$ and $34.9 \mathrm{ng}$ per $100 \mathrm{ml}$, respectively). Abdominal computed tomography showed marked atrophy of the left kidney (Figure 1a). Computed tomography angiography further revealed complete occlusion of the left renal artery, whereas blood flow of the right renal artery was intact (Figure 1b). The ${ }^{99 \mathrm{~m}} \mathrm{Tc}$-mercaptoacetyltriglycine renography also revealed the functional loss of the atrophic left kidney in contrast to the preserved right renal function with blood supply (Figure 1c). After the diagnosis of RVH complicated with nephrotic syndrome, treatment with an ARB, valsartan ( $80 \mathrm{mg}$ per day), was commenced. Following a 3-week treatment period with valsartan, the patient's proteinuria was slightly reduced ( $\sim 3 \mathrm{~g}$ per day), but his blood pressure was still high at $\sim 170 / 90 \mathrm{~mm} \mathrm{Hg}$. Eplerenone (25 mg per day), a selective MR blocker, was administered in addition to the ARB, calcium channel blockers and an alpha blocker that were also being taken by the patient (Figure 2). After 6 months of commencement of treatment with eplerenone at $50 \mathrm{mg}$ per day, the systemic blood pressure was normalized to $\sim 130 / 70 \mathrm{~mm} \mathrm{Hg}$, and the concomitant proteinuria was reduced to $\sim 1.0 \mathrm{~g}$ per day (Figure 2). The serum albumin level increased $(3.8 \mathrm{~g}$ per $100 \mathrm{ml})$ and the total cholesterol level decreased $(183 \mathrm{mg}$ per $100 \mathrm{ml})$, whereas the RAA activity (plasma renin activity, $>20 \mathrm{ng} \mathrm{ml}^{-1} \mathrm{~h}^{-1}$; aldosterone concentration, $12.4 \mathrm{ng}$ per $100 \mathrm{ml}$ ) was not greatly changed. During the course of eplerenone treatment, no adverse effects including hyperkalemia and progress of renal damage (24-h creatinine clearance, $50 \mathrm{ml} \mathrm{min}^{-1}$ ) were observed.

The present case showed hypertension and renal insufficiency caused by an uncommon complication of RVH with nephrotic syndrome. The patient who had a high RAA activity was additionally treated with an ARB and an MR blocker, leading to the improvement of hypertension and the successful reduction of proteinuria. Takahashi et al. ${ }^{2}$ reported a similar case of RVH complicated with nephrotic syndrome that was successfully treated with an ARB, candesartan. Considering the multiple renal actions of angiotensin II, including vasoconstriction, cell proliferation, activation of local growth factors and stimulation of adrenal aldosterone release, the massive proteinuria in RVH is possibly caused by non-hemodynamic RAA actions on glomeruli, leading to hyperfiltration in addition to angiotensin II's hemodynamic actions. Based on the results of a study by Halimi et al., patients with nephroticrange proteinuria associated with renovascular

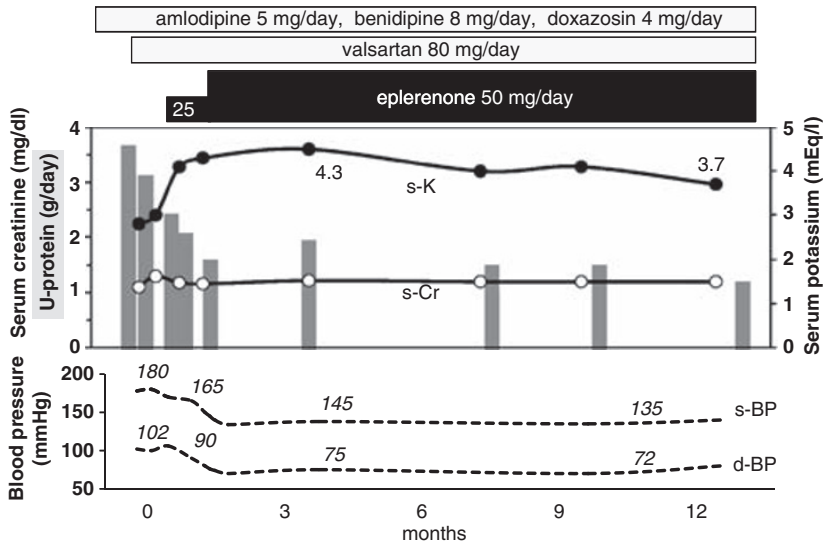

Figure 2 Clinical course: changes in the levels of daily urinary protein (U-protein: gray bars), serum creatinine levels (s-Cr: open circles), serum potassium levels (s-K: filled circles) and systolic (s-BP) and diastolic blood pressure (d-BP) are shown.

diseases are likely to have common characteristics, including an age over 50 years, a smoking habit, atherosclerotic vascular complications, hypokalemia and complete obstruction of renal arteries. ${ }^{3}$

The mechanism of nephrotic-range proteinuria with RVH remains unclear; however, cases in which histological examination was performed have revealed a characteristic pathology of focal segmental glomerulosclerosis in the contralateral kidney. ${ }^{4-7}$ In such cases, massive proteinuria has been successfully reduced by the administration of an ACEI and/or an ARB, by nephrectomy or renal angioplasty. ${ }^{2,8}$ These findings suggest that excess activation of the RAA system may be critical for accelerating proteinuria in $\mathrm{RVH}$ patients. In recent years, aldosterone has also been recognized as a major risk factor for damage to various organs, including the cardiovascular system, brain and kidneys. Similar to angiotensin II, aldosterone also contributes to the progression of renal injury through hemodynamic and direct cellular actions via MR. ${ }^{9}$ Blockage of aldosterone actions has been shown to be effective for reducing proteinuria in patients with chronic kidney disease who had already been treated with an ACEI and/or an ARB in a clinical randomized study. ${ }^{9,10}$ The selectivity of proteinuria is known to be highly correlated with the severity of tubulointerstitial damage in nephrotic syndrome, and thus the selectivity index is useful for predicting the development and progression of proteinuric nephropathy. ${ }^{1}$ In the present case, the patient had a moderately selective level of the selectivity index, which may indicate less severity of the tubulointerstitial damage in the remaining kidney, resulting in the preservation of renal function after combined therapy with an ARB and an MR blocker. Kuriyama et al. also reported that treatment with eplerenone in addition to an ACEI and an ARB effectively reduced proteinuria in a chronic kidney disease patient with nephrotic-range proteinuria. ${ }^{8}$ In the present case, antiproteinuric and antihypertensive effects were added to the preceding antihypertensive effects derived from drugs including an ARB by the addition of eplerenone administration. This add-on effect may have been elicited by the efficacious reduction of renal MR activity by eplerenone in combination with the preceding effects of an ARB.

In summary, this is the first case of RVH in which eplerenone successfully reduced concomitant nephrotic proteinuria, possibly by the suppression of renal MR actions. Because activation of the RAA system has a key role in the progression of renal injury in $\mathrm{RVH}$, combination therapy including eplerenone and an ACEI and/or an ARB could be useful for ameliorating proteinuria due to $\mathrm{RVH}$, with careful attention to serum potassium levels and the function of the remaining kidney.

Jiro Suzuki ${ }^{1}$, Fumio Otsuka ${ }^{1}$, Kenichi Inagaki ${ }^{1}$, Katsuyuki Tanabe ${ }^{1}$, Naoko Tsukamoto ${ }^{1}$, Tomoko Miyoshi ${ }^{1}$, Eri Nakamura ${ }^{1}$, Toshio Ogura ${ }^{1}$, Isao Kumagai ${ }^{2}$ and Hirofumi Makino ${ }^{1}$

${ }^{1}$ Department of Medicine and Clinical Science, Okayama University Graduate School of Medicine, Dentistry and Pharmaceutical Sciences, Okayama, Japan and ${ }^{2}$ Teraoka Memorial Hospital, Fukuyama, Japan

E-mail: fumiotsu@md.okayama-u.ac.jp 
1 Bazzi C, Petrini C, Rizza V, Arrigo G, D’Amico G. A modern approach to selectivity of proteinuria and tubulointerstitial damage in nephrotic syndrome. Kidney Int 2000; 58: 1732-1741.

2 Takahashi F, Hasebe N, Chinda J, Okada M, Takeuchi T, Hirayama T, Imamoto C, Kikuchi K. A case of nephrotic syndrome associated with renovascular hypertension successfully treated with candesartan. Hypertens Res 2003; 26: 123-127.

3 Halimi JM, Ribstein J, Du Cailar G, Mimran A. Nephrotic-range proteinuria in patients with renovascular disease. Am J Med 2000; 108: 120-126.

4 Alchi B, Shirasaki A, Narita I, Nishi S, Ueno M, Saeki T, Miyamura S, Gejyo F. Renovascular hypertension: a unique cause of unilateral focal segmental glomerulosclerosis. Hypertens Res 2006; 29: 203-207.

5 Cote I, Boucher D, Lebel M. Renovascular hypertension and focal segmental glomerulosclerosis in the contralateral kidney. J Hum Hypertens 2001; 15: 143-144.

6 Fujii S, Kasahara H, Oguchi H. [A case of renovascular hypertension with nephrotic syndrome, accompanied by focal segmental glomerulosclerosis-like lesion in the contralateral kidney]. Nippon Jinzo Gakkai Shi 1991; 33: 1017-1024.

7 Ubara Y, Hara S, Katori H, Yamada A, Morii H. Renovascular hypertension may cause nephrotic range proteinuria and focal glomerulosclerosis in contralateral kidney. Clin Nephrol 1997; 48: 220-223.
8 Kuriyama S, Sugano N, Ueda H, Otsuka $\mathrm{Y}$, Kanzaki G, Hosoya T. Successful effect of triple blockade of renin-angiotensin-aldosterone system on massive proteinuria in a patient with chronic kidney disease. Clin Exp Nephrol 2009; 13: 663-666.

9 Nishiyama A, Hitomi $H$, Rahman A, Kiyomoto $H$. Drug discovery for overcoming chronic kidney disease (CKD): pharmacological effects of mineralocorticoidreceptor blockers. J Pharmacol Sci 2009; 109: $1-6$.

10 Bianchi S, Bigazzi R, Campese VM. Long-term effects of spironolactone on proteinuria and kidney function in patients with chronic kidney disease. Kidney Int 2006; 70: 2116-2123. 